\title{
Petrographic-geochemical characterization of the dispersed organic matter in Menilite shales from the Silesian Unit in the Carpathian Mountains of SE Poland
}

\begin{abstract}
This article is devoted to the issue of dispersed organic matter present in Menilite shales. A total of 27 samples collected from 8 outcrops, representing various lithological types (mainly shales and mudstones) types, were examined with the use of microscopic (optical microscopy) and geochemical (Rock-Eval pyrolysis) methods. Maceral composition in investigated rocks is relatively monotonous. Liptinite macerals are usually the most numerous, while vitrinite macerals are less common. Inertinite macerals are very rare. The maceral composition suggest brackish environment. Results of both the vitrinite reflectance and the Rock-Eval pyrolysis show that the maturity level of the organic matter changes from the immature to the oil window phase. Maturity increases towards the south-east.
\end{abstract}

Key words: Menilite shales, organic matter, vitrinite reflectance, liptinite, Rock-Eval pyrolysis.

\section{Charakterystyka petrograficzno-geochemiczna rozproszonej materii organicznej w łupkach menilitowych z jednostki śląskiej w obrębie polskiej części Karpat Zewnętrznych}

\begin{abstract}
Artykuł poświęcony jest zagadnieniu rozproszonej materii organicznej. Przy użyciu metod mikroskopowych (mikroskopia optyczna w świetle przechodzącym i odbitym) i geochemicznych (piroliza Rock-Eval) przebadano ogółem 27 próbek pobranych z 8 odsłonięć, reprezentujących różne typy litologiczne, głównie różnego rodzaju łupki i mułowce. Przebadane skały charakteryzują się stosunkowo monotonnym składem macerałowym. Dominują macerały grupy liptynitu, mniej licznie obserwuje się macerały grupy witrynitu, natomiast grupa inertynitu jest obecna jedynie incydentalnie. Skład macerałowy sugeruje środowisko brakiczne. Zarówno wyniki pomiarów refleksyjności witrynitu, jak i pirolizy Rock-Eval wskazują, iż stopień dojrzałości materii organicznej jest zmienny i waha się od stadium niedojrzałego po główne okno ropne, przy czym obserwuje się trend wzrostowy w kierunku południowo-wschodnim.
\end{abstract}

Słowa kluczowe: łupki menilitowe, materia organiczna, refleksyjność witrynitu, liptynit, piroliza Rock-Eval.

\section{Introduction}

The Menilite Beds are one of the most interesting formations in the Carpathian Mountains, due to their diverse lithology, occurrence and dispersed organic matter content (its composition and maturity). These features have a great impact when considering them as potential source rocks and for that reason much attention is paid to them [2, 4, 6-17].

The aim of this article is to characterize the organic matter present in the Menilite shales (both its composition and maturity level). Menilite Beds have a complex lithology.
Different types of shales (clay, marly, carbonate), as well as siltstones, mudstones, sandstones and cherts occur in the outcrops. This paper investigates whether the lithological character of rocks, influences the variability of the organic matter composition. The relationship of organic matter with basic geochemical parameters obtained from Rock-Eval pyrolysis (TOC, HI, $\mathrm{T}_{\max }$ ) was also analyzed. The article focuses on the Menilite Beds from the eastern part of the Silesian unit (figure 1). 


\section{Research material and methods}

The research material consisted of 27 samples taken from 8 outcrops within the Silesian Unit (Polish part of the Carpathian Mountains). These outcrops were: Rudawka Rymanowska, Rymanów Zdrój, Wernejówka, Bóbrka, Jabłonki, Strzebowiska, Wetlina and Monasterzec (figure 1). Strzebowiska, Jabłonki and Wetlina are located in the Fore-Dukla zone, which is the southern part of the Silesian Unit. During the field work, special attention was paid to collecting samples that represent all the possible lithological varieties that can be distinguished in the outcrop scale. This was to examine how the dispersed organic matter composition changes within each outcrop and also, what are the relations of organic matter composition and the lithology of the samples. The lack of such information would prevent a fair comparison of samples taken from the various outcrops. The applied selection of the outcrops allows to extrapolate obtained results in order to determine trends of changes in the composition and maturity of the analyzed organic matter in a regional scale. The collected samples represented mainly gray shales (with or without carbonates), while siltstones, mudstones and cherts were rather rare.

The analyses were based mostly on microscopic observations. They were divided into two stages. The first stage involved the analysis in reflected light and also in fluorescence mode, with the use of polished sections. They were performed on a Zeiss Axioplan optical microscope, at a magnification of $500 \times$, in immersion oil (immersol $518 \mathrm{~N}, \mathrm{n}=1.518$ ).

Firstly, a point-counting method was performed (min. 600 points were counted). It was done to examine both the amount and ratio between macerals of the three main groups - vitrinite, inertinite and liptinite. Secondly, the measurements of the vitrinite reflectance were performed. Before performing the measurements, the microscope was calibrated with the use of two reflection standards $\left(R_{o}=0.429\right.$ spinel, garnet $\left.R_{o}=0.905\right)$.

The second stage was performed in transmitted light, with the use of thin sections that were analyzed with the Nikon Eclipse LV100 POL optical microscope, at magnifications $50 \div 1000 \times$. The point-counting method was used again, this time to determine the type of rock and its mineral composition (min. 500 points were counted).

The results obtained during the work on both microscopes were supplemented with photographic documentation.

Microscopic analyses were also supplemented with the results of the Rock-Eval pyrolysis, performed on the RockEval - 6 apparatus (standard model). Among the obtained parameters, special attention was paid to TOC (Total Organic Carbon), $T_{\max }$ and HI (Hydrogen Index), as these are very important in organic matter characterization.

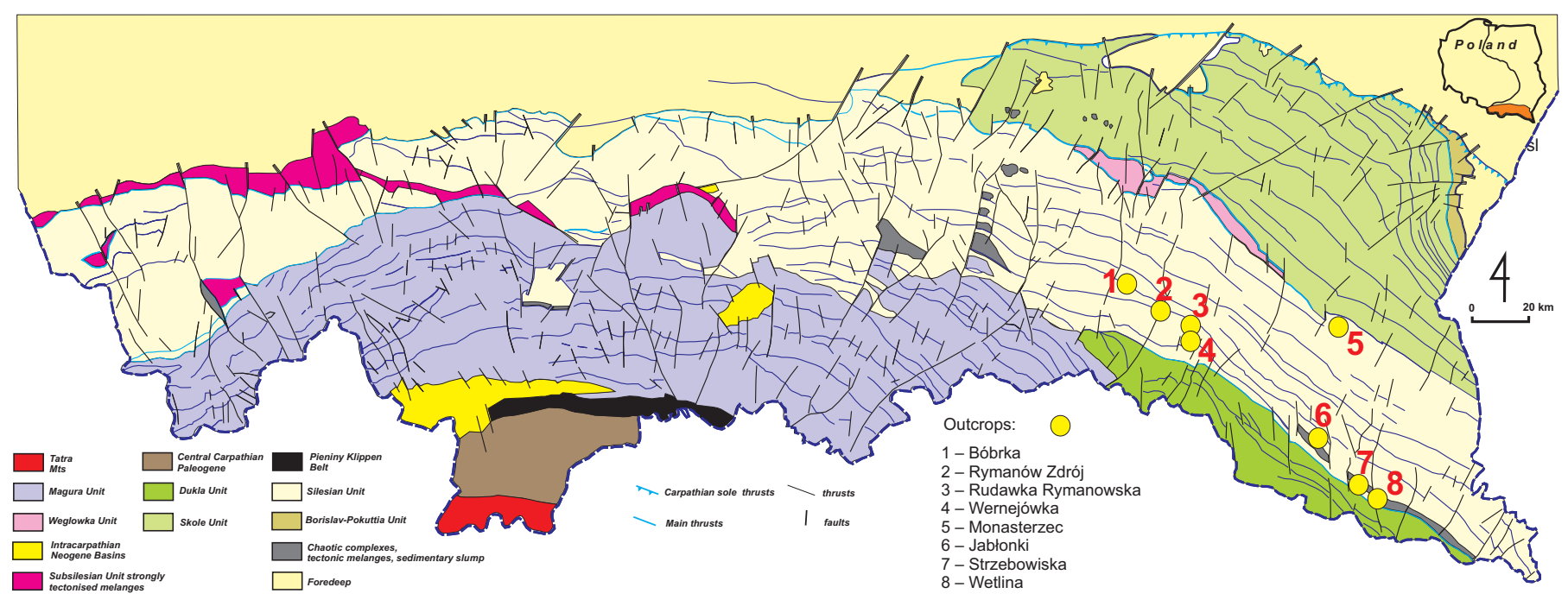

Fig. 1. Geological map of the investigated area with the position of the outcrops [3, edited]

\section{Results}

The maceral composition of the investigated samples is relatively monotonous. Analyzed rocks differ mainly in maceral proportions. Macerals of all three main groups (vitrinite, inertinite and liptinite) are present in the examined rocks (tab. 1). In some samples solid bitumen were present.
The solid bitumen usually exhibit orange-yellow fluorescence (plate I, F).

Liptinite macerals are the most numerous organic components observed in analyzed samples (up to $33.3 \%$ vol., average $9.7 \%$ vol.), represented by alginite, bituminite and liptodetrinite. 


\begin{tabular}{|c|c|c|c|c|c|c|c|c|c|c|c|c|c|c|c|c|c|c|c|c|c|c|c|c|c|c|}
\hline \multirow{4}{*}{ 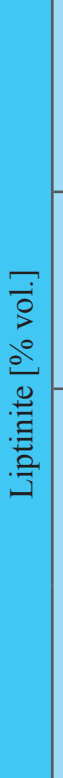 } & \multicolumn{2}{|c|}{ 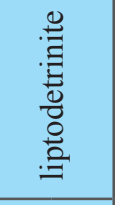 } & $\begin{array}{l}n \\
\tilde{b} \\
i \\
\end{array}$ & $\begin{array}{c}\mathcal{T} \\
\dot{m}\end{array}$ & $\stackrel{\vec{i}}{i}$ & $\hat{\sigma}$ & $\stackrel{\tilde{g}}{\stackrel{r}{r}}$ & 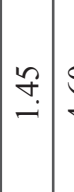 & $\begin{array}{l}8 \\
\dot{+} \\
+\end{array}$ & $\mid \begin{array}{l}\stackrel{2}{a} \\
\dot{f}\end{array}$ & $\stackrel{\overbrace{}}{\unlhd}$ & \begin{tabular}{c|c}
+ & $i$ \\
$\infty$ & 4 \\
$i$ & $c$
\end{tabular} & $\begin{array}{l}\infty \\
n \\
i\end{array}$ & $\begin{array}{l}\mathscr{E} \\
\mathbb{E} \\
\mathbb{E}\end{array}$ & 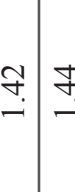 & $\begin{array}{l}+ \\
\dot{v}\end{array}$ & 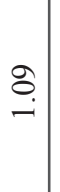 & \begin{tabular}{c|c}
$\mathscr{\Xi}$ & $\mathscr{\Xi}$ \\
$\Xi$ & $\Xi$
\end{tabular} & 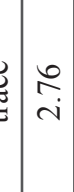 & $\stackrel{\cap}{-}$ & 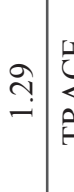 & 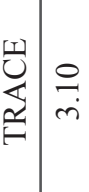 & 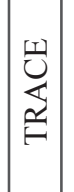 & \begin{tabular}{l|l}
$\stackrel{8}{0}$ & $\vdots$ \\
$i$ &
\end{tabular} & $\stackrel{\oplus}{\oplus}$ & : \\
\hline & \multicolumn{2}{|c|}{ 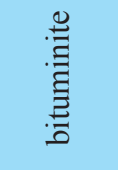 } & $\hat{m}$ & f. & $\mid \begin{array}{l}\stackrel{2}{*} \\
\stackrel{+}{+}\end{array}$ & $\hat{\tilde{o}}$ & $\begin{array}{l}\mathscr{E} \\
\Xi \\
\Xi\end{array}$ & $\underset{⿱}{+}$ & $\begin{array}{l}\infty \\
n \\
0\end{array}$ & त) & 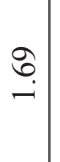 & 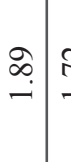 & $\stackrel{N}{I}$ & \begin{tabular}{l|l}
$\mathscr{E}$ \\
$\mathbb{E}$ & $\bar{z}$
\end{tabular} & \begin{tabular}{l|l}
$\stackrel{n}{=}$ & $\infty$ \\
$=$ & $\infty$
\end{tabular} & 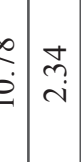 & $\begin{array}{l}\hat{n} \\
\infty \\
\infty\end{array}$ & $\begin{array}{l}\mathscr{\Xi} \\
\Xi \\
\Xi\end{array}$ & 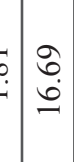 & m̂n & 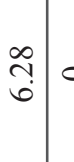 & ○ $\underset{\text { f }}{\stackrel{f}{-}}$ & ?. & $\stackrel{\overbrace{}}{\stackrel{r}{n}}$ & ભે & †্ \\
\hline & \multirow{2}{*}{ 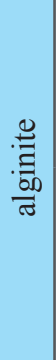 } & 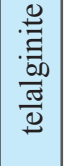 & $\frac{n}{0}$ & \begin{tabular}{|l|} 
\\
$\mathscr{\Xi}$ \\
$\Xi$
\end{tabular} & \begin{tabular}{|l|} 
\\
$\mathscr{\Xi}$ \\
$\Xi$ \\
\end{tabular} & $\begin{array}{l}\mathscr{\Xi} \\
\mathscr{E} \\
\Xi\end{array}$ & 0 & $\begin{array}{lll}0 & 0\end{array}$ & 0 & 0 & 0 & \begin{tabular}{c|c}
0 \\
$\circ$ \\
0
\end{tabular} & 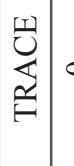 & 0 & $\begin{array}{lll}\mathscr{E} & 0 \\
\mathbb{E} & 0\end{array}$ & 00 & 0 & 0 & 0 & 0 & 00 & 0 & 0 & $\begin{array}{l}0 \\
0 \\
0\end{array}$ & $\begin{array}{l}\mathscr{U} \\
\mathbb{E} \\
\Xi\end{array}$ & $\begin{array}{l}\mathscr{E} \\
\mathscr{E} \\
\mathbb{E}\end{array}$ \\
\hline & & 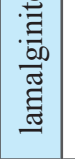 & $\begin{array}{l}\infty \\
i n \\
i n \\
\end{array}$ & 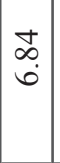 & $\begin{array}{l}\infty \\
0 \\
0 \\
0\end{array}$ & $\begin{array}{c}8 \\
i \\
i\end{array}$ & $\begin{array}{l}\infty \\
\vec{\sim} \\
\end{array}$ & \begin{tabular}{c|c}
1 & \\
0 & $\vdots$ \\
0 &
\end{tabular} & 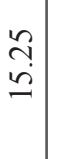 & $\begin{array}{c}0 \\
\infty \\
0 \\
\varrho\end{array}$ & $\stackrel{\overbrace{}}{-}$ & \begin{tabular}{ll}
$\circ$ & $=$ \\
\hdashline
\end{tabular} & $\begin{array}{c}7 \\
\dot{v} \\
i\end{array}$ & \begin{tabular}{l|l}
$\mathscr{E}$ \\
$\stackrel{\Xi}{\Xi}$
\end{tabular} & \begin{tabular}{c|c}
2 & $\tilde{\sigma}$ \\
$\hat{\sigma}$ & 0 \\
0
\end{tabular} & $\begin{array}{l}c \\
0 \\
0\end{array}$ & $\begin{array}{l}0 \\
0 \\
0\end{array}$ & \begin{tabular}{c|c}
$\overrightarrow{0}$ & $\stackrel{0}{0}$ \\
\end{tabular} & $\vec{b} \overrightarrow{\dot{b}}$ & 0 & 0 & \begin{tabular}{l|l}
$\mathscr{\Xi}$ \\
$\mathbb{\Xi}$ & $\stackrel{\Xi}{\Xi}$
\end{tabular} & 0 & $\hat{n}$ & $\begin{array}{l}\bar{n} \\
\dot{s} \\
n\end{array}$ & ले \\
\hline \multirow{3}{*}{ 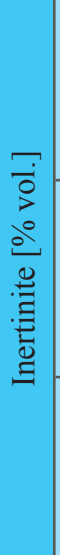 } & $\Rightarrow$ & 递 & $\begin{array}{l}\mathscr{E} \\
\mathbb{\Xi}\end{array}$ & \begin{tabular}{|l|}
0 \\
$\mathscr{\Xi}$ \\
$\Xi$
\end{tabular} & $\begin{array}{l}\mathscr{8} \\
\mathbb{E} \\
\mathbb{E}\end{array}$ & $\begin{array}{l}\mathscr{O} \\
\mathbb{E} \\
\Xi\end{array}$ & 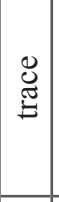 & $\stackrel{\mathscr{E}}{\mathscr{E}}$ & 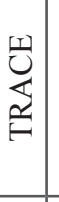 & $\begin{array}{l}n \\
0 \\
0\end{array}$ & $\begin{array}{l}\mathscr{\Xi} \\
\mathbb{\Xi}\end{array}$ & $\begin{array}{l}\mathscr{\Xi} \\
\mathbb{\Xi} \\
\Xi\end{array}$ & $\begin{array}{l}\mathscr{E} \\
\stackrel{\Xi}{\Xi}\end{array}$ & 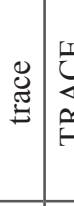 & 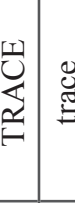 & $\begin{array}{l}\mathbb{E} \\
\mathbb{E} \\
\mathbb{E}\end{array}$ & 0 & $\circ \mid$\begin{tabular}{l|l}
$\mathscr{U}$ \\
$\mathscr{\Xi}$
\end{tabular} & 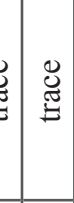 & 0 & $0 \mid c$ & \begin{tabular}{l|l}
0 & $\mathscr{E}$ \\
$\Xi$
\end{tabular} & $\begin{array}{l}\mathscr{Z} \\
\overparen{\Xi} \\
\mathbb{E}\end{array}$ & $\begin{array}{l}\mathscr{\Xi} \\
\mathbb{\Xi}\end{array}$ & $\begin{array}{l}\mathscr{E} \\
\mathbb{\Xi}\end{array}$ & $\mathbb{\Xi}$ \\
\hline & 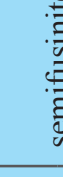 & 鄫 & $\begin{array}{l}\mathscr{E} \\
\ddot{\Xi}\end{array}$ & \begin{tabular}{|l|}
0 \\
$\mathscr{E}$ \\
$\mathscr{\Xi}$
\end{tabular} & $\begin{array}{l}\mathscr{E} \\
\mathscr{E}\end{array}$ & 0 & $\begin{array}{l}0 \\
\mathscr{U} \\
\Xi \\
\Xi\end{array}$ & \begin{tabular}{l|}
$\mathscr{J}$ \\
$\mathscr{\Xi}$ \\
$\Xi$
\end{tabular} & 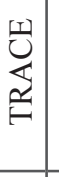 & $\begin{array}{l}\mathscr{\Xi} \\
\mathscr{\Xi}\end{array}$ & $\begin{array}{l}\mathscr{\Xi} \\
\mathbb{\Xi}\end{array}$ & $\begin{array}{l}\mathscr{\Xi} \\
\underset{\Xi}{\Xi}\end{array}$ & 0 & 0 & \begin{tabular}{l|l}
$\mathscr{E}$ & $\mathscr{E}$ \\
$\Xi$ & $\mathscr{\Xi}$
\end{tabular} & $\begin{array}{l}\mathscr{E} \\
\mathbb{E} \\
\mathbb{E}\end{array}$ & 0 & ○| & $\begin{array}{l}B_{0} \\
E\end{array}$ & 0 & 0 & \begin{tabular}{l|l}
$\mathscr{U}$ \\
$\mathbb{E}$
\end{tabular} & $\begin{array}{l}\mathscr{E} \\
\mathbb{\Xi}\end{array}$ & $\begin{array}{l}\mathscr{\Xi} \\
\mathbb{\Xi} \\
\Xi\end{array}$ & $\begin{array}{l}\mathscr{E} \\
\mathbb{\Xi}\end{array}$ & o \\
\hline & 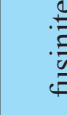 & & 0 & $\begin{array}{l}\mathscr{U} \\
\mathscr{E} \\
\end{array}$ & \begin{tabular}{|l|} 
\\
$\mathscr{E}$ \\
$\mathbb{E}$
\end{tabular} & $\begin{array}{l}\mathscr{\Xi} \\
\mathbb{\Xi} \\
\end{array}$ & $\begin{array}{l}\mathscr{\Xi} \\
\dddot{\Xi}\end{array}$ & 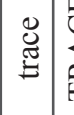 & 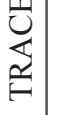 & $\underset{\mathscr{\Xi}}{\mathscr{\Xi}}$ & 0 & \begin{tabular}{l|l}
$\stackrel{\mathscr{\Xi}}{\Xi}$ \\
$\stackrel{\Xi}{\Xi}$
\end{tabular} & $\circ$ & $\underset{\Xi}{\mathscr{\Xi}}$ & \begin{tabular}{l|l}
$\mathscr{\Xi}$ & $\mathscr{E}$ \\
$\Xi$ & $\mathscr{\Xi}$
\end{tabular} & 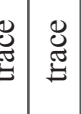 & 0 & ○| & $\begin{array}{lll}3 & 0 \\
\end{array}$ & 0 & 0 & ○ & $\stackrel{0}{0}$ & $\begin{array}{l}\mathscr{\Xi} \\
\overparen{\Xi} \\
\end{array}$ & $\begin{array}{l}\stackrel{\mathscr{E}}{\mathbb{\Xi}} \\
\stackrel{\Xi}{|c|}\end{array}$ & o \\
\hline \multirow{3}{*}{ 总 } & \multicolumn{2}{|c|}{$\begin{array}{l}\text { 兽 } \\
\text { 总 } \\
\text { 总 }\end{array}$} & 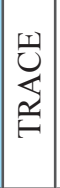 & 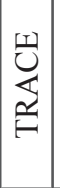 & $\begin{array}{l}0 \\
0 \\
0\end{array}$ & 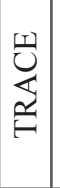 & 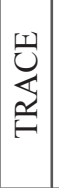 & J্ & $\stackrel{?}{f}$ & $\begin{array}{l}2 \\
0 \\
0\end{array}$ & 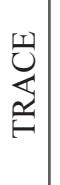 & 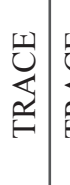 & 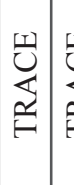 & 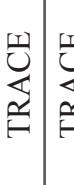 & 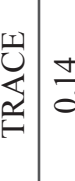 & 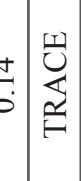 & 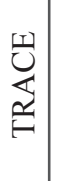 & $\circ \mid$ & 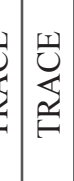 & \begin{tabular}{l|}
$\mathscr{\Xi}$ \\
$\mathbb{\Xi}$ \\
\end{tabular} & $\underset{\Xi}{\mathscr{\Xi}}$ & \begin{tabular}{l|l}
$\stackrel{0}{+}$ & 0 \\
\hdashline & $\stackrel{0}{0}$
\end{tabular} & 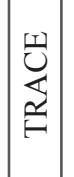 & 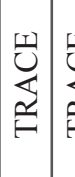 & 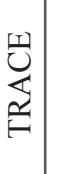 & 㱟 \\
\hline & \multirow[t]{2}{*}{ : } & 总 & $\begin{array}{l}\mathscr{E} \\
\mathscr{\Xi} \\
\end{array}$ & $\mid \begin{array}{l}\mathscr{U} \\
\dddot{\Xi}\end{array}$ & $\begin{array}{l}\mathscr{E} \\
\dddot{\Xi}\end{array}$ & $\begin{array}{l}\mathscr{\Xi} \\
\mathscr{\Xi}\end{array}$ & $\begin{array}{l}\mathscr{E} \\
\Xi \\
\Xi\end{array}$ & $\begin{array}{l}\mathscr{\Xi} \\
\dddot{\Xi} \\
\end{array}$ & $\begin{array}{l}\mathscr{E} \\
\mathbb{E}\end{array}$ & 0 & 0 & 0 & 0 & 0 & \begin{tabular}{l|l}
$\mathscr{E}$ & $\mathscr{E}$ \\
$\Xi$
\end{tabular} & $\begin{array}{l}\mathbb{E} \\
\mathbb{E}\end{array}$ & 0 & 0.0 & 0 & 0 & 0 & 0 & 0 & 0 & 0 & $\begin{array}{l}\mathscr{\Xi} \\
\mathbb{\Xi}\end{array}$ \\
\hline & & $\begin{array}{l}\text { 音 } \\
\overline{0} \\
0 \\
\overline{0} \\
\end{array}$ & $\stackrel{-}{a}$ & 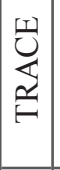 & 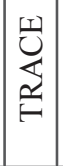 & 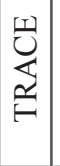 & $\begin{array}{l}0 \\
0 \\
\circ\end{array}$ & $\stackrel{n}{0}$ & $\stackrel{?}{\stackrel{f}{\circ}}$ & 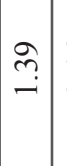 & $\begin{array}{l}0 \\
\stackrel{0}{0} \\
0\end{array}$ & 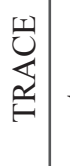 & $\underset{\Xi}{\mathscr{E}}$ & \begin{tabular}{l|l}
$\mathscr{E}$ \\
$\Xi$ \\
$\Xi$
\end{tabular} & \begin{tabular}{l|l}
$\frac{1}{0}$ \\
\\
0 & $\frac{1}{0}$
\end{tabular} & 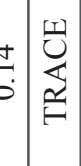 & $\begin{array}{l}\mathscr{\Xi} \\
\mathbb{\Xi}\end{array}$ & $\circ \mid$ & 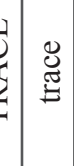 & $\mid \begin{array}{l}\mathscr{\Xi} \\
\Xi \\
\Xi\end{array}$ & \begin{tabular}{c|c}
0 \\
-1 \\
0
\end{tabular} & 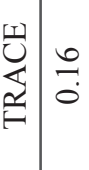 & 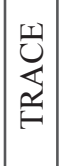 & ণे & $\stackrel{n}{\circ}$ & कै \\
\hline & 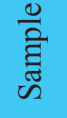 & & $\begin{array}{l}\stackrel{+}{\sim} \\
\stackrel{0}{\circ}\end{array}$ & $\begin{array}{c}2 \\
a \\
0 \\
0 \\
-\end{array}$ & 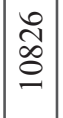 & $\begin{array}{l}\hat{A} \\
0 \\
0 \\
=\end{array}$ & 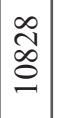 & 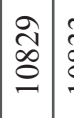 & $\begin{array}{l}m \\
\tilde{O} \\
0 \\
0\end{array}$ & 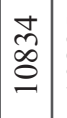 & $\begin{array}{l}\approx \\
\approx \\
\infty \\
0\end{array}$ & \begin{tabular}{l|l}
$\vec{f}$ & \\
$\stackrel{0}{0}$ & $\vdots$ \\
\hdashline
\end{tabular} & 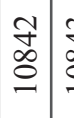 & 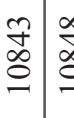 & 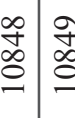 & 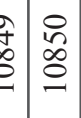 & $\stackrel{\stackrel{\Im}{\Xi}}{\beth}$ & 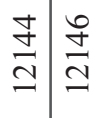 & 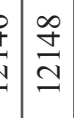 & 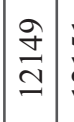 & 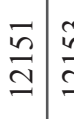 & \begin{tabular}{l|l}
$\stackrel{n}{a}$ & $n$ \\
& $\stackrel{n}{\beth}$
\end{tabular} & $\begin{array}{l}\tilde{n} \\
\stackrel{n}{\beth}\end{array}$ & $\begin{array}{l}\infty \\
\stackrel{\infty}{\beth} \\
\stackrel{\Xi}{\beth}\end{array}$ & 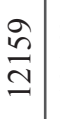 & $\frac{\mathfrak{d}}{\sqrt{2}}$ \\
\hline & $\begin{array}{l}\stackrel{0}{0} \\
\text { 0. } \\
0 \\
0\end{array}$ & & & & 告 & 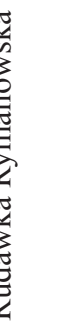 & & & & 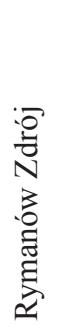 & & & 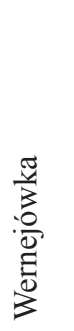 & & 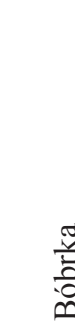 & 邆 & & $\begin{array}{l}\frac{\pi}{0} \\
\frac{0}{0} \\
\frac{0}{0}\end{array}$ & & 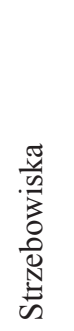 & & 营 & & & 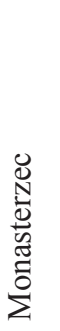 & \\
\hline
\end{tabular}


Alginite content varies from trace to $30.5 \%$ vol. (average $4 \% \mathrm{vol}$.). This is mainly lamalginite, present in the form of single algae bodies or algae colonies (plate I, A). Apart from lamalginite, telalginite is also (but rarely) observed $(<0.2 \%$ vol.). Telalginite is present in a form of a single algae with relatively thick cell walls, which most likely can be classified as Tasmanites (plate I, B). Both types of alginite exhibit a strong, bright yellow fluorescence. Another very common maceral within the liptinite group is bituminite (plate I, C). Bituminite is present usually in the form of laminae. It generally shows brownish fluorescence, but in a few samples no fluorescence was observed. Bituminite content in analyzed samples reaches up to $18.6 \%$ vol. (average $4.2 \%$ vol.). In all the samples the fine liptinite detritus $(<10 \mu \mathrm{m})$, most likely of algal origin, is noticed (average content is $2.3 \%$ vol., but it can reach up to $5.6 \%$ vol.). Liptodetrinite exhibits yellow fluorescence.

Apart from liptinite, macerals of the vitrinite group are also present in the investigated rocks. In the analyzed samples both collotelinite and detrovitrinite are common, while telinite is rare. Telovitrinite reaches the maximum content of $1.4 \%$ vol. (average $0.2 \%$ vol.), while detrovitrinite does not exceed $0.5 \%$ vol. There are various types of vitrinite particles present (plate I, D). Some grains have reduced reflectance, due to saturation with lipoid substance. They are usually present in the form of elongated clasts (tens of microns) and exhibit brown fluorescence. Next to them, elongated particles of a slightly higher reflectance, in which the lipoid impregnation is less intense, are present. Finally, redeposited vitrinite particles with the highest reflectance can be distinguished. The diversity of reworked vitrinite reflectance values may suggest its origin from various sources. They usually have an oval shape and do not show any fluorescence. Apart from the grains described above, very rarely, grains of telinite with preserved cellular structure can be noticed. Grains of detrovitrinite are relatively common. They occur in the form of small $(<10 \mu \mathrm{m})$, sharp-edged particles.

The least common macerals in the analyzed samples are inertinite macerals. They are present in trace amounts, only rarely approaching $0.2 \%$ vol. There are fragments of fusinite, semifusinite and inertodetrinite present. Fusinite is characterized by the highest reflectance and preserved cellular structure (usually a few cells). Semifusinite has a lower reflectance than fusinite, with a similar state of preservation of the morphological features. There are also particles of fusinite and semifusinite where the cell structure is not preserved, but the characteristic shape of sharp-edged grains can still be seen (plate I, E). Small $(<10 \mu \mathrm{m})$ fragments of inertodetrinite are relatively often, but their content is rather very low.

\section{Thermal maturity}

The commonly used parameter of thermal maturity is vitrinite reflectance $\left(R_{o}\right)$. In case of the analyzed samples this parameter is in the range of 0.24 to $0.94 \%$ (table 2), which indicates the maturity spectrum of the analyzed rocks from immature (17 samples) to the oil window phase (6 samples). For four samples it was impossible to provide reliable measurements of the vitrinite reflectance, due to a very low number of measurements. In these samples both the size and surface quality of the vitrinite particles were not suitable for the analysis. The obtained reflectance measurements are confirmed by the results of Rock-Eval pyrolysis (table 2). The $T_{\max }$ parameter is in the range of 412 to $468^{\circ} \mathrm{C}$, corresponding to both immature (16 samples) and the oil window (11 samples) stage of maturity.

The organic matter content (TOC parameter) in the case of the analyzed samples is in the range from 0.3 to $10.4 \%$ (average 3.4\%). Hydrogen index (HI) also changes in a wide range of 16 to 597, which mainly corresponds to types II and III of kerogen and also to their mixture (figure 2).

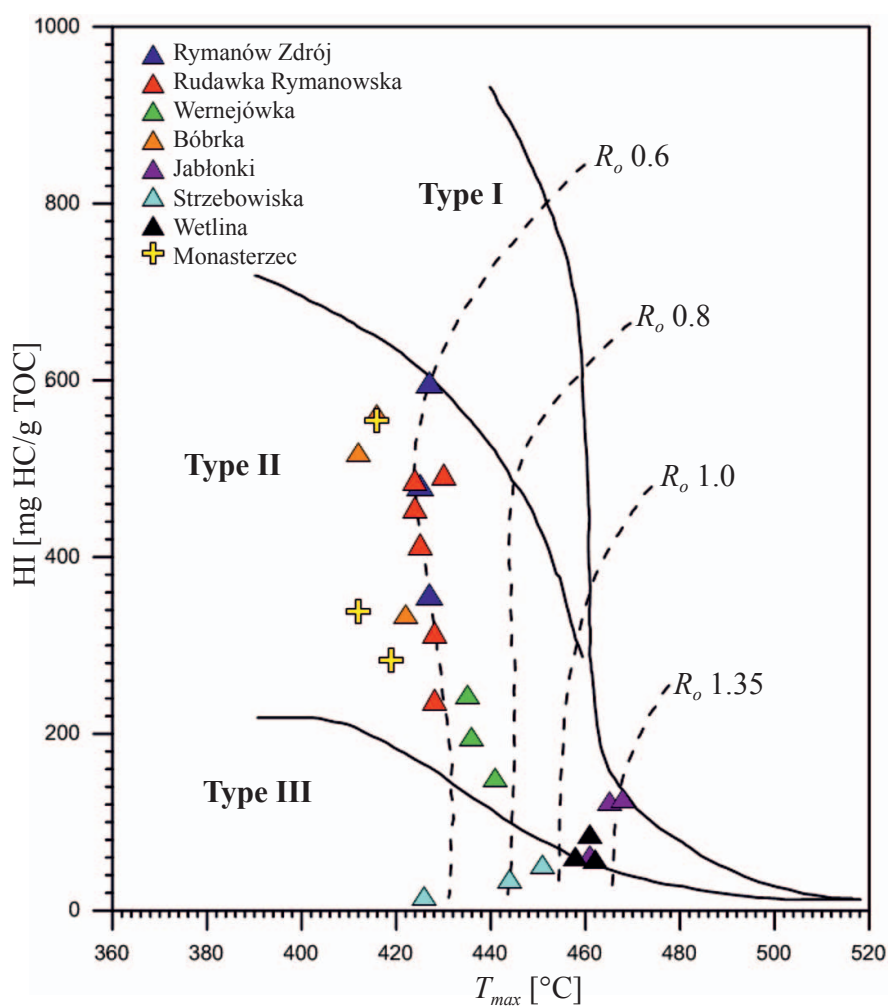

Fig. 2. The analyzed samples plotted into van Krevelen's HI- $T_{\max }$ diagram

\section{The relations between dispersed organic matter content and lithology}

The composition of organic matter in relation to the type of rock dispersed within it, is an interesting issue. Unfortunately, such characteristics in this work cannot go beyond only very general observations. This is due to two reasons. Firstly, in the analyzed samples, the content of both vitrinite 
Table 2. Vitrinite reflectance measurement results and the most important geochemical parameters obtained for analyzed samples

\begin{tabular}{|c|c|c|c|c|c|c|c|}
\hline \multirow{2}{*}{ Outcrop } & \multirow{2}{*}{ Sample } & \multirow{2}{*}{ Lithology } & \multirow{2}{*}{$\begin{array}{l}T_{\max } \\
{\left[{ }^{\circ} \mathrm{C}\right]}\end{array}$} & \multirow{2}{*}{$\begin{array}{l}\text { TOC } \\
{[\%]}\end{array}$} & \multirow{2}{*}{ HI } & \multicolumn{2}{|c|}{$R_{o}[\%]$} \\
\hline & & & & & & mean & std. dev. \\
\hline \multirow{6}{*}{ Rudawka Rymanowska } & 10824 & clay shale & 424 & 6.57 & 456 & 0.24 & 0.07 \\
\hline & 10825 & clay shale & 424 & 4.05 & 488 & 0.31 & 0.08 \\
\hline & 10826 & clay shale & 425 & 4.35 & 415 & 0.30 & 0.05 \\
\hline & 10827 & mudstone & 428 & 1.29 & 239 & 0.29 & 0.06 \\
\hline & 10828 & clay shale & 428 & 2.97 & 314 & 0.24 & 0.05 \\
\hline & 10829 & mudstone & 430 & 2.84 & 493 & 0.26 & 0.03 \\
\hline \multirow{3}{*}{ Rymanów Zdrój } & 10833 & mudstone & 427 & 7.08 & 597 & 0.25 & 0.05 \\
\hline & 10834 & mudstone & 425 & 10.42 & 481 & 0.26 & 0.07 \\
\hline & 10835 & clay shale & 427 & 3.34 & 358 & 0.27 & 0.06 \\
\hline \multirow{3}{*}{ Wernejówka } & 10841 & clay shale & 436 & 2.06 & 197 & $\mathrm{x}$ & $\mathrm{x}$ \\
\hline & 10842 & clay shale & 435 & 2.55 & 244 & 0.33 & 0.07 \\
\hline & 10843 & siltstone & 441 & 0.82 & 151 & 0.35 & 0.11 \\
\hline \multirow{3}{*}{ Bóbrka } & 10848 & clay shale & 416 & 6.82 & 562 & 0.28 & 0.09 \\
\hline & 10849 & mudstone & 412 & 5.05 & 518 & 0.26 & 0.05 \\
\hline & 10850 & clay shale & 422 & 3.12 & 336 & 0.29 & 0.08 \\
\hline \multirow{3}{*}{ Jabłonki } & 12143 & marly shale & 465 & 3.81 & 124 & 0.75 & 0.13 \\
\hline & 12144 & chert & 468 & 0.35 & 126 & $\mathrm{x}$ & $\mathrm{x}$ \\
\hline & 12146 & marly shale & 461 & 0.32 & 62 & $\mathrm{x}$ & $\mathrm{x}$ \\
\hline \multirow{3}{*}{ Strzebowiska } & 12148 & clay shale & 444 & 2.87 & 36 & 0.72 & 0.08 \\
\hline & 12149 & clay shale & 451 & 1.45 & 52 & $\mathrm{x}$ & $\mathrm{x}$ \\
\hline & 12151 & mudstone & 426 & 2.78 & 16 & 0.77 & 0.06 \\
\hline \multirow{3}{*}{ Wetlina } & 12153 & carbonate shale & 462 & 0.78 & 58 & 0.94 & 0.15 \\
\hline & 12155 & clay shale & 461 & 2.03 & 87 & 0.83 & 0.08 \\
\hline & 12157 & carbonate shale & 458 & 0.70 & 61 & 0.94 & 0.11 \\
\hline \multirow{3}{*}{ Monasterzec } & 12158 & clay shale & 412 & 4.84 & 339 & 0.28 & 0.07 \\
\hline & 12159 & clay shale & 416 & 4.17 & 554 & 0.24 & 0.05 \\
\hline & 12162 & clay shale & 419 & 3.40 & 283 & 0.26 & 0.05 \\
\hline
\end{tabular}

and inertinite maceral groups, is usually very low, effectively making it impossible to examine the statistically reliable relationship between them, in relation to the different lithologies. Secondly, the group of collected samples, although relatively diverse in terms of their lithology (different varieties of shales, siltstones, mudstones, chert, limestone), is too small for all rock types to be represented in an amount, where it could be possible to conclude that the obtained results are characteristic for the specific lithological type. Having that in mind, it can only be stated that:

- average content of the dispersed organic matter in analyzed shales and mudstones is in the range of from 1 to $5 \%$ vol. and is significantly higher than in the studied siltstone and chert, where it does not exceed $1 \%$ vol.,

- average content of vitrinite in all types of rocks does not exceed $0.6 \%$ vol.; the largest content is observed for the clay shales, carbonate shales and mudstones $(1 \div 1.6 \%$ vol.), while in the marly shales and siltstone, only trace amount of vitrinite is present; vitrinite is not observed in the studied chert, - inertinite is usually very rare - it is observed most frequently in carbonate shales and mudstones, while in chert it is absent,

- content of liptinite is usually the highest; it is observed most often in clay shales, marly shales and mudstones (average of $11 \div 12 \%$ vol.); the content of this maceral group in the carbonate shales is slightly lower (approximately $2 \%$ vol.), while in the siltstone and chert it does not exceed $0.7 \%$ vol.

Data for the maceral groups with respect to lithology is shown in figure 3 . The separate results for alginite, bituminite and liptodetrinite, due to their high content in the analyzed samples, are also presented. 


\section{PLATE I}
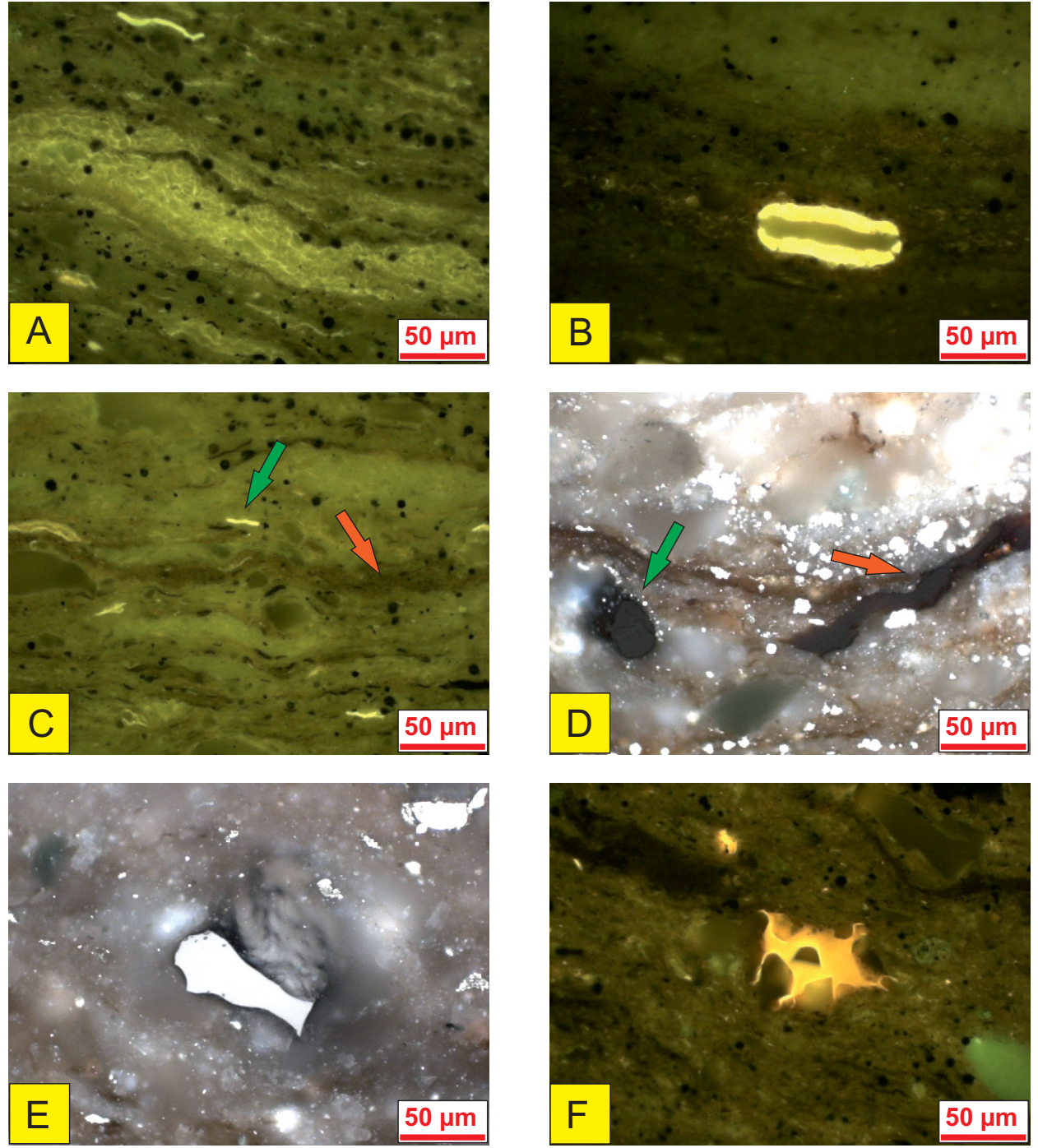

A - Lamalginite. Monasterzec, sample 12159.

B - Telalginite. Wernejówka, sample 10842.

C - Bituminite (red arrow) and alginite (green arrow). Bóbrka, sample 10849.

D - Two kinds of vitrinite particles elongated, not reworked (red arrow) and oval, reworked (green arrow). Rudawka Rymanowska, sample 10827.

E - Fusinite. Jabłonki, sample 12146.

F - Solid bitumen. Strzebowiska, sample 12149.

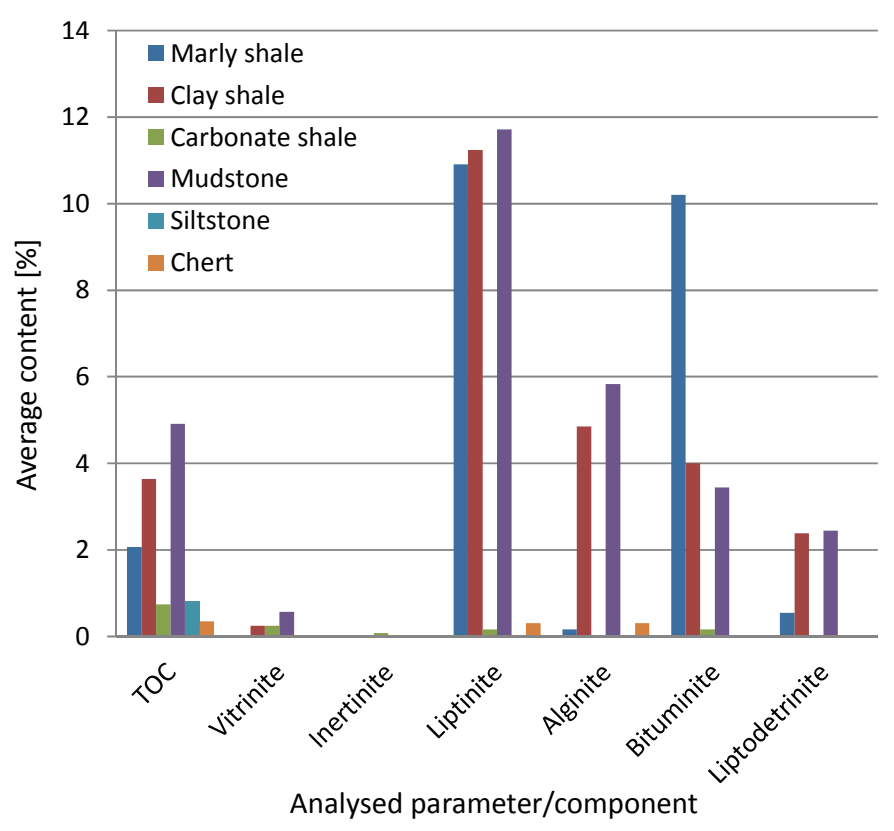

Fig. 3. Average content of different macerals and maceral groups in relation to the lithology
Correlations of the most important geochemical parameters, vitrinite reflectance and organic matter composition

Correlations between the most important parameters obtained from the Rock-Eval analysis, results of the vitrinite reflectance measurements and liptinite maceral group content were analyzed. There were a number of interesting relations noticed (table 3 ), of which the most important are:

- $\quad$ very good correlation (correlation index $=0.84$ ) of $T_{\max }$ and $R_{o}$ parameters; these two parameters define the maturity of organic matter,

- strong correlation $(-0.78)$ between vitrinite reflectance $\left(R_{o}\right)$ and the hydrogen index, which is reasonable, because the HI decreases with the increasing degree of organic matter transformation; consequently, the hydrogen index also decreases with the increasing $T_{\max }$ parameter $(-0.69)$,

- the presence of alginite has a significant impact on the hydrogen index (0.58), which should not be surprising, as alginite is assigned to the types I and II of kerogen (which 
have a high hydrogen index); lack or a lower content of the alginite in the studied rocks lowers the HI parameter down to values characteristic for the type III kerogen (at low or even trace content of vitrinite, which is the true type III of kerogen).

\section{The maturity of organic}

\section{matter and its regional variability}

The results from both vitrinite reflectance measurements and the analysis of Rock-Eval (parameter $T_{\max }$ ), allowed to determine regional trends in the changes of organic matter

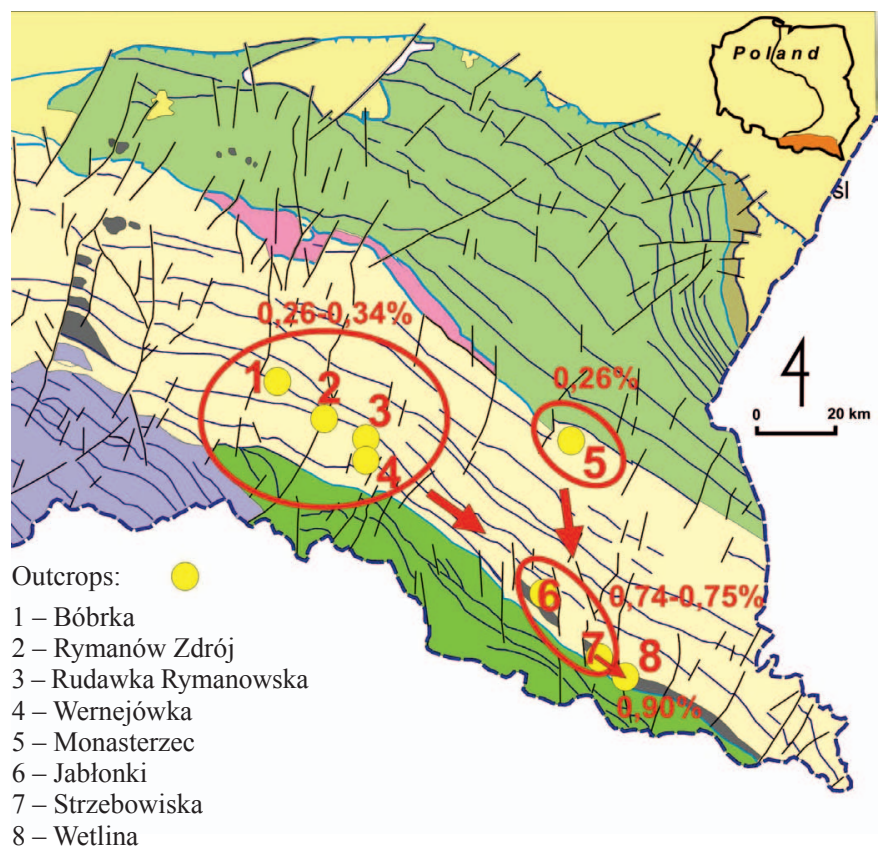

Fig. 4. Vitrinite reflectance changes within the analyzed area
Table 3. Correlations between different parameters and maceral components

\begin{tabular}{|l|r|r|r|r|r|r|c|}
\cline { 2 - 8 } \multicolumn{1}{c|}{} & $R_{o}$ & $T_{\max }\left[{ }^{\circ} \mathrm{C}\right]$ & TOC $[\%]$ & HI & Liptinite & Alginite & Bituminite \\
\hline$R_{o}$ & & & & & & & \\
\hline$T_{\max }\left[{ }^{\circ} \mathrm{C}\right]$ & 0.84 & & & & & & \\
\hline OOC $[\%]$ & -0.50 & -0.62 & & & & & \\
\hline $\mathrm{HI}$ & -0.82 & -0.76 & 0.75 & & & & \\
\hline Liptinite & -0.28 & -0.45 & 0.66 & 0.54 & & & \\
\hline Alginite & -0.43 & -0.46 & 0.49 & 0.63 & 0.77 & & \\
\hline Bituminite & 0.22 & -0.02 & 0.22 & -0.09 & 0.48 & -0.15 & \\
\hline Liptodetrinite & -0.34 & -0.37 & 0.71 & 0.47 & 0.45 & 0.36 & -0.04 \\
\hline
\end{tabular}

maturity. The increase maturity level from immature in the western and northern parts of the analyzed area to the oil window phase in the southern-east part was noticed. This trend is shown in figures 4 and 5 .

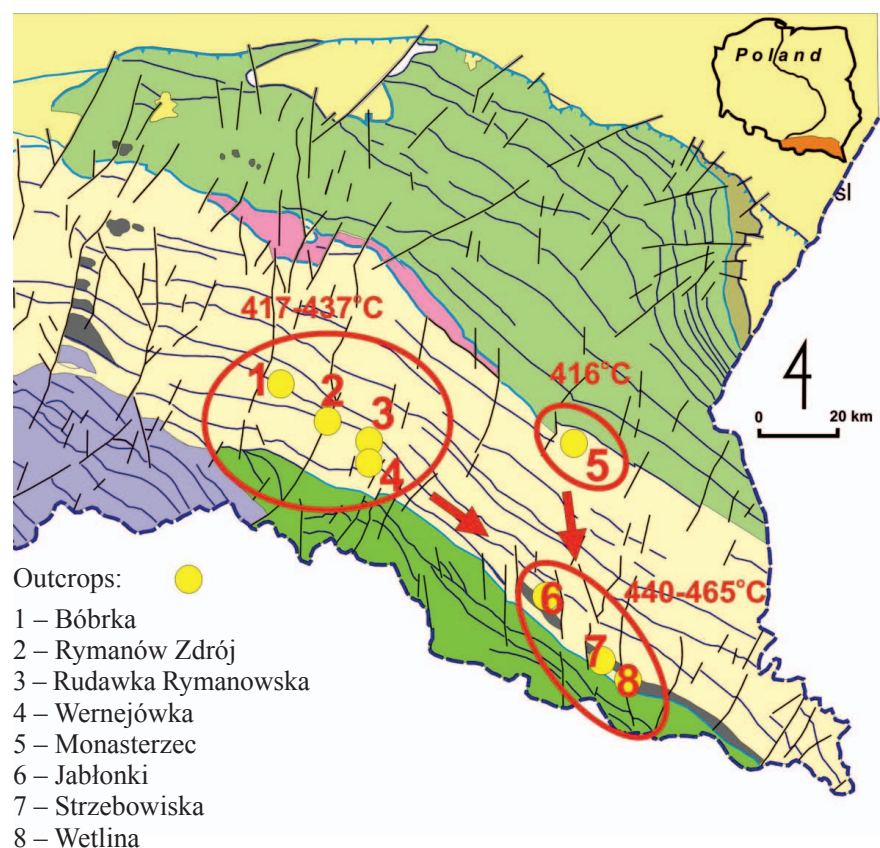

Fig. 5. The $T_{\max }$ parameter distribution within the analyzed area

\section{Conclusions}

1. Maceral composition of the investigated samples is relatively monotonous - analyzed rocks differ mainly in maceral proportions. Liptinite macerals (alginate, bituminite and liptodetrinite) are the most numerous. Vitrinite macerals (collotelinite, telinite, detrovitrinite) are common, but their content is lower than liptinite macerals. Inertinite macerals (fusinite, semifusinite and inertodetrinite) are rather rare.

2. There is an increase maturity level from immature in the western and northern parts of analyzed area to the oil window phase in the southern-east part.

3. Analyzed shales and mudstones are significantly richer in organic components than studied siltstone and chert.
Liptinite is most numerous in clay shales, marly shales and mudstones, while the highest content of vitrinite is in clay and carbonate shales and also in mudstones.

4. There are several good correlations between organic matter components described with the use of optical microscope and geochemical parameters obtained from Rock-Eval pyrolysis. These two methods used together can give very detailed and complete information on organic matter components in rocks.

5. The maceral association (alginie + bituminie + vitrinite + detritus of all 3 main maceral groups) present in the samples may suggest the brackish environment [1]. 
Please cite as: Nafta-Gaz 2017, no. 11, pp. 835-842, DOI: 10.18668/NG.2017.11.02

Article contributed to the Editor 13.01.2017. Approved for publication 6.07.2017.

This paper is based on the results from statutory work entitled: Characteristics of the dispersed organic matter in menilite shales from Silesian Unit within the Polish part of the Carpathian Mts - archive no. SG-4101-7/16.

\section{Literature}

[1] Bousein B., Stein R.: Black shale formation in the late Paleocene/early Eocene Arctic Ocean and paleoenvironmental conditions: New results from a detailed organic petrological study. Marine and Petroleum Geology 2009, vol. 26, s. 416-426.

[2] Curtis J.B., Kotarba M.J., Lewan M.D., Więcław D.: Oil/ source rock correlations in the Polish Flysch Carpathians and Mesozoic basement and organic facies of the Oligocene Menilite Shales: insights from hydrous pyrolisis experiments. Organic Geochemistry 2004, vol. 35, pp. 1573-1596.

[3] Jankowski L., Kopciowski R., Ryłko W. (red.): Geological map of the Outer Carpathians: boreland of Ukraine and Romania. 1:200 000. Państwowy Instytut Geologiczny, Warszawa 2007.

[4] Koltun Y.V.: Organic matter in Oligocene Menilite Formation rocks of the Ukrainian Carpathians: palaeoenvironment and geochemical evolution. Organic Geochemistry 1992, vol. 18, s. $423-430$.

[5] Kosakowski P., Więcław D., Kotarba M.J.: Charakterystyka macierzystości wybranych utworów fliszowych w przygranicznej strefie polskich Karpat Zewnętrznych. Geologia 2009, vol. 35, pp. 155-190.

[6] Köster J., Kotarba M., Lafargue E., Kosakowski P.: Source rock habitat and hydrocarbon potential of Oligocene Menilite Formation (Flysch Carpathians, Southeast Poland): an organic geochemical and isotope approach. Organic Geochemistry 1998, vol. 29, pp. 543-558.

[7] Köster J., Rospondek M., Schouten S., Kotarba M., Zubrzycki A., Sinninghe Damste J.S.: Biomarker geochemistry of a foreland basin: Oligocene Menilite Formation in the Flysch Carpathians of Southeast Poland. Organic Geochemistry 1998, vol. 29, pp. 649-669.

[8] Kotarba M.J., Więcław D., Koltun Y.V., Kuśmierek J., Marynowski L., Dudok I.V.: Organic geochemical study and genetic correlation of natural gas, oil and Menilite source rocks in the San and Stryi rivers region (Polish and Ukrainian Carpathians). Organic Geochemistry 2007, vol. 38, pp. 1431-1456.

[9] Kotarba M.J., Więcław D., Koltun Y.V., Lewan M.D., Marynowski L., Dudok I.V.: Organic geochemical study and genetic correlations between the Oligocene Menilite source rocks and oil and natural gas from surface seepages and from deep accumulations in the Starunia area (Ukrainian Carpathians). [W:] Kotarba, M.J. (ed.): Polish and Ukrainian geological survey in 2004-2005 years in Starunia at the area of finds of woolly rhinoceroses and other extinct vertebrates. Polish Geological Institute and "Geosphere", WarszawaKraków 2005, pp. 125-146.
[10] Lewan M.D., Kotarba M.J., Curtis J.B., Więcław D., Kosakowski, P.: Oil-generation kinetics for organic facies with Type-II and-IIS kerogen in the Menilite Shales of the Polish Carpathians. Geochimica et Cosmochimica Acta 2006, vol. 70, pp. 3351-3368.

[11] Matyasik I.: Badania geochemiczne warstw menilitowych, inoceramowych i spaskich jednostki skolskiej fliszu karpackiego. Nafta-Gaz 1994, vol. 40, pp. 234-243.

[12] Matyasik I., Kupisz L.: Geological and geochemical conditions of the hydrocarbon generation in the Menilite Beds from the south part of the Strzyżów Depression. The $2^{\text {nd }}$ Conference on the Geochemical and Petrophysical Investigations in Oil and Gas Exploration, Janowice 10-12.04.1996, pp. 179-197.

[13] Mroczkowska-Szerszeń M., Ziemianin K., Brzuszek P., Matyasik I., Jankowski L.: The organic matter type in the shale rock samples assessed by FTIR-ATR analyses. Nafta-Gaz 2015, no. 6, pp. 361-369.

[14] Rospondek M.J., Köster J., Sinninghe Damsté J.S.: Novel $C_{26}$ highly branched isoprenoid thiophenes and alkane from the Menilite Formation, Outer Carpathians, SE Poland. Organic Geochemistry 1997, vol. 26, pp. 295-304.

[15] Więcław D.: Geneza oligoceńskiej ropy naftowej polskich Karpat fliszowych - siarka organiczna w kerogenie warstw menilitowych a kinetyka procesu generowania węglowodorów. Rozprawa doktorska, Akademia Górniczo-Hutnicza, Kraków 2002.

[16] Więcław D., Kotarba M.J., Koltun Y.: Identification of kerogen type in the menilite shales of the Skole and Silesian units of the Polish and Ukrainian Carpathians. $23^{\text {rd }}$ International Meeting on Organic Geochemistry, Torquay (England) 09-01.09.2007, P22-M.

[17] Ziemianin K., Brzuszek P., Słoczyński T., Jankowski L.: Dispersesd organic matter in shales from Menilite Beds within Polish Outer Carpathians - preliminary diagnosis. Nafta-Gaz 2015, no. 9, pp. 615-623.

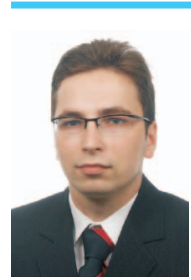
Mgr Konrad ZIEMIANIN

Asystent w Zakładzie Geologii i Geochemii. Instytut Nafty i Gazu - Państwowy Instytut Badawczy ul. Lubicz 25 A

31-503 Kraków

E-mail: konrad.ziemianin@inig.pl 